\title{
Barriers to Utilization of HIV Care Services Among Adolescents and Young Adults in Rakai, Uganda: the Role of Economic Strengthening
}

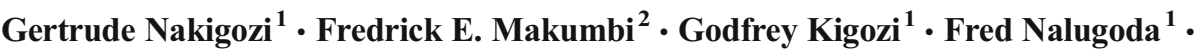 \\ Steven J. Reynolds ${ }^{3,4,5}$ - Larry W. Chang ${ }^{4,5}$ - Joseph Kagaayi ${ }^{1}$ - David Serwadda ${ }^{2}$. \\ Maria J. Wawer ${ }^{5}$ Ronald H. Gray ${ }^{5}$
}

Published online: 1 May 2015

(C) Springer International Publishing 2015

\begin{abstract}
Background Early entry into HIV care is low in Sub-Saharan Africa. In Rakai, adolescents and young adults 15-24 years were at increased risk of not entering care after knowledge of their positive HIV status. This qualitative study explored barriers to entry into care from HIV-positive clients aged 1524 years and explored the role of economic strengthening in promoting care utilization.

Methods We conducted 18 in-depth interviews among HIVinfected individuals aged 15-24 years, who had not entered care within 6 months of result receipt and referral for free care. Also, key informant interviews were conducted with 12 providers. Interviews were audio-recorded and transcripts subjected to thematic content analysis based on the health belief model.

Results Barriers to using HIV care included fear of stigma (7/18-39\%) and HIV disclosure and high transport costs. Programmatic barriers included fear of antiretroviral drug side effects, long waiting, and travel times. Denial of HIV status
\end{abstract}

Gertrude Nakigozi

gnakigozi@rhsp.org

Rakai Health Sciences Program, Kalisizo, Uganda

2 School of Public Health, Makerere University College of Health Sciences, Kampala, Uganda

3 Division of Intramural Research, National Institute of Allergy and Infectious Diseases, National Institutes of Health, Bethesda, MD, USA

4 Division of Infectious Diseases, Department of Medicine, Johns Hopkins School of Medicine, Baltimore, MD, USA

5 Department of Epidemiology, Johns Hopkins Bloomberg School of Public Health, Baltimore, MD, USA and absence of AIDS symptoms were also barriers. Eight (44\%) of the participants reported economic barriers to care utilization, and these included lack of money for transport to clinic and anticipated lost work time and money resulting from long waiting clinic hours.

Conclusion Economic barriers are highly prevalent as obstacles to utilization of care by young people, suggesting the role of economic strengthening for young persons living with HIV, so as to promote utilization of HIV care services.

Keywords HIV · Adolescents · Young adults · HIV care utilization · Economic barriers $\cdot$ Economic strengthening

\section{Introduction}

Sub-Saharan Africa (SSA) remains the most heavily affected region by HIV/AIDS, contributing at least two thirds $(67 \%)$ of the global 35.3 million cases and $70 \%$ of the 2.3 million global annual new infections (UNAIDS 2013). In 2012, $7.2 \%$ of Uganda's population was living with HIV and this amounts to an estimated 1.5 million people, of which 100,000 were children under 15 years (UNAIDS 2013). An estimated 63, 000 people died from AIDS in 2012 (UNAIDS 2013), and 1.1 million children have been orphaned by Uganda's devastating epidemic (UNAIDS 2012). In 2012, new infections in Uganda were estimated at 140,000 (2013) and 20,000 are among children under 15 years.

There is a need for all HIV-positive persons to access care and preventive interventions in a timely manner (UNAIDS 2013; Mermin et al. 2006; Graham et al. 2010; Granich et al. 2010) to prevent morbidity and mortality (Amuron et al. 2009; Losina et al. 2010; Bassett et al. 2009). However, uptake of free HIV care services after diagnosis 
remains suboptimal. A study in Uganda showed that $31.5 \%$ of HIV-positive persons who knew their HIV status had not enrolled into care 6 months after testing HIV positive (Nakigozi et al. 2011). Similar findings have been observed in the USA (Samet et al. 1998; Althoff et al. 2010; Fagan et al. 2010; Marks et al. 2010). Also, adolescents and young people (ages 15-24 years) were at a significantly higher risk of not utilizing HIV care services after knowledge of their positive HIV status compared to other age groups (Nakigozi et al. 2011)

For antiretroviral therapy (ART) to be effective and for infected persons not to develop drug resistance, adherence to the treatment regimen must be followed. Recognized barriers to ART have included poverty, immobility, deteriorating memory, and poor comprehension of complex treatment by elderly care takers (Skovdal et al. 2011), and food insecurity has been associated with missed clinic visits (Weiser et al. 2012).

Another study examining allocation of household resources for health care showed that resources were allocated differently between age groups. Households allocated significantly fewer resources to the health care of sick children compared to that of sick adults, with the rationale to concentrate its resources spent for health care on productive members rather than to spread them equitably among all its sick members (Sauerborn et al. 1996). The allocation could be worse among HIV-infected youths, since most of the young people infected with HIV are orphans being looked after by other families (Kamali et al. 1996).

We set out to describe barriers to utilization of HIV care and treatment and to explore the role of economic barriers in preventing service use among adolescents and young adults who had failed to enter care 6 months after referral for free HIV care in Rakai, Uganda. This study was part of a larger study examining non-enrollment and non-adherence to HIV care and barriers to utilization of HIV care in Rakai, Uganda.

\section{Methods}

Study Design This was a qualitative study nested within a study examining barriers to utilization of free HIV care in Rakai, Uganda (Nakigozi et al. 2013).

Study Setting and Participants Study participants were drawn from the Rakai community Cohort Study (RCCS), an approximately annual surveillance of consenting/assenting persons aged 15 to 49 years in 50 villages in rural Rakai district, South Western Uganda. The RCCS has close to 1400 HIV-infected participants, the majority of whom (over $90 \%$ ) know their HIV status and have received counseling and referral (Nakigozi et al. 2011). All RCCS participants who provided blood for HIV testing had free access to HIV counseling either as individuals or couples by trained Rakai program counselors. HIV-positive individuals who accepted their HIV results received posttest counseling and education on the need to enroll for HIV care and were referred to the Rakai Program HIV/AIDS (Suubi or "hope") clinic closest to their residence.

\section{Sampling and Data Collection}

Key informant participants were purposively sampled from among consenting/assenting adolescents and young adults aged 15-24 years, who had received a positive HIV diagnosis but had not enrolled for HIV care within 6 months after referral for free HIV services. These were actively traced in the community by HIV counselors, using the address given at their most recent RCCS interview. Interviewers were acquainted with the study geographical locations and, hence, able to trace addresses of participants. When in doubt, they utilized the services of community field guides. Participant tracing and interview continued until the intended sample size of 18 was complete. Those interviewed were selected based on availability at time of home visit by the counselor and willingness/ability to provide written informed consent/ assent prior to the interview. Twenty-three of 30 attempts to locate participants were successful. Of the 23 , two individuals refused to participate due to lack of time for the interview and three had no guardian available to provide written informed consent (Fig. 1).

Between September 2010 and January 2011, we conducted 18 in-depth interviews (IDIs) among consenting/assenting clients aged 15 to 24 years, who had received their HIV diagnosis but had not enrolled for HIV care within 6 months after referral for free HIV services. Twelve key informant

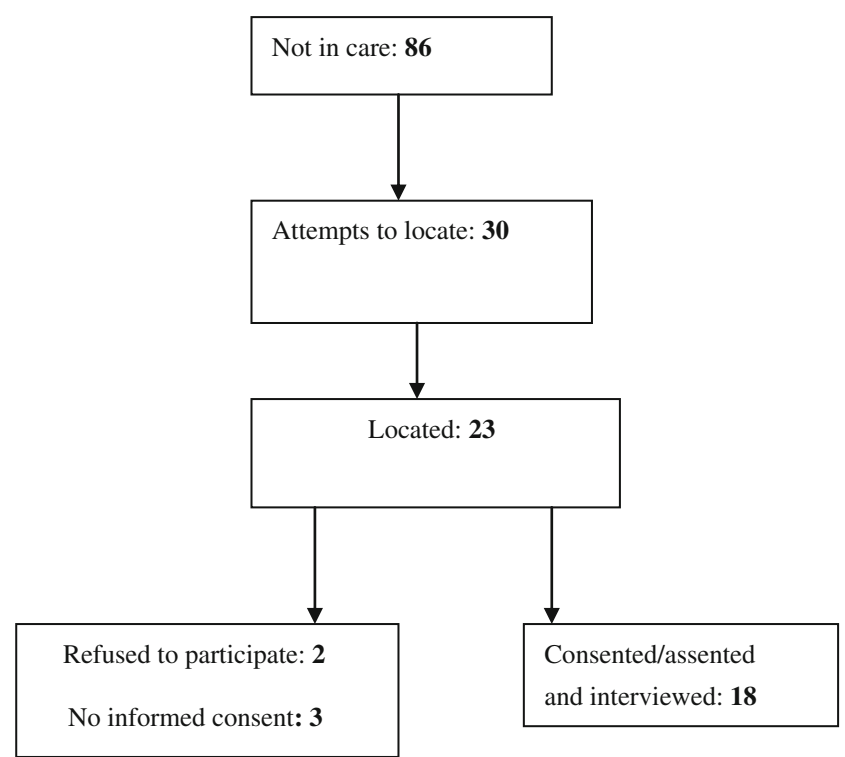

Fig. 1 Consort diagram 
interviews (KIIs) were conducted with health care providers (three HIV counselors, three home visitor nurses, and six peer educators). Informed consent/ assent and permission to record the interview were obtained from all participants. Parents or guardians of participants aged 15-17 years provided informed consent in addition to the participants' assent to participate in the study. Interviews were conducted by two trained research assistants (29-year-old female and 40-year-old male) with experience in qualitative data collection (additional 2-day training in data collection for this study was provided). Interviews were conducted in Luganda (the most commonly spoken local language in Rakai). IDIs lasted approximately $1 \mathrm{~h}$ and KIIs about $40 \mathrm{~min}$. All interviews were audio-recorded and transcribed.

\section{Data Management and Analysis}

All interviews were transcribed then translated into English by the interviewer. Transcript review began while data collection was still underway, and participant responses were used to shape future interview questions. Verbatim transcripts were used for analysis. Two independent coders used the word-processed text to develop a codebook by categorizing responses from the interviews to identify themes generated from the in-depth and KIIs about barriers to enrollment into HIV care. The coded passages were collapsed into categories and grouped in accordance with the health belief model (HBM) to conceptualize determinants of failure to enroll into HIV care services. These HBM concepts included perceived susceptibility to HIV, perceived severity of HIV disease, and perceived barriers to enrollment for care.

The HBM The health belief model (HBM) is a psychological model that attempts to explain and predict health behaviors, by focusing on the attitudes and beliefs of individuals. The basic structure of the model is derived from psychological theories suggesting that health-seeking behaviors are determined at the individual level by a desire to avoid illness and a belief that a particular course of action will prevent or relieve illness (Janz and Becker 1984). The HBM uses constructs representing the perceived threat and net benefits such as perceived susceptibility, severity, benefits, barriers, cues to action (the concept of "readiness to act"), and self-efficacy or confidence in the ability to successfully perform an action. The model has previously been used to describe clinic attendance, as well as HIV-related behaviors among various populations including those in African settings (Mkanta and Uphold 2006). In this study, the HBM was applied to conceptualize the individual and social determinants of enrollment into care among un-enrolled HIV-infected individuals.

\section{Ethical Considerations}

Approval for the study was obtained from the Makerere University School of Public Health Higher Degrees Research and Ethics Committee and the Uganda National Council of Science and Technology. All study participants were informed of study procedures, risks, and benefits and provided written consent/assent for enrollment. Research assistants were well trained in the conduct of qualitative data collection and the ethical issues in human research. Participants received unique study numbers, and data sets contained no personal identifiers. Data was stored in password-protected computers accessible only by authorized personnel. IDI and KII participants were assured of confidentiality, and each interview was conducted in private. Participants received additional counseling to enroll for HIV care.

\section{Results}

Informant Characteristics In the previous study examining non-enrollment into care, 86 out of the 163 $(52.8 \%)$ persons aged 15-24 did not enroll into care. For this qualitative study, 18 of the non-enrolled participants were successfully contacted and completed indepth qualitative interviews. Of the 18 , nine were male and nine female, $12(67 \%)$ were married, and 8 were peasant farmers. Only $7 / 18(39 \%)$ had postprimary education while one had no formal education. Twelve HIV care providers (three HIV counselors, three home visitor nurses, and six peer educators) provided key informant information.

\section{Perceived Barriers to Utilizing HIV Care Services}

Stigma, defined as a social process, experienced or anticipated, characterized by exclusion, rejection, blame, or devaluation that results from experience, perception, or anticipation of an adverse social judgment about a person or group (Weiss et al. 2006) emerged as a barrier to utilization of HIV care services among seven (39\%) of the participants. In these participants, stigma manifested as expressions of fear of being seen by other people at the HIV clinic, hence unintentionally disclosing their HIV status. Participants anticipated disrespect, ill treatment, or social isolation by their peers or the community, if known to be HIV infected, as shown by the quotes below.

I feared. I thought that if I go for care and others see me, they will begin to despise me, they will stop giving me respect. (IDI, Male, 23) 
You know it was a big determination to go for the HIV test something I actually did. However, what caused trouble was the fear I developed. To be seen by my fellow young men at the clinic and you hear them say out there in the community that "a young man like you go to the clinic for septrin," so I felt hesitant to go to the clinic. They may say, what were you looking for to get "HIV"; such words belittle me and I totally gave up. (IDI, Male, 20)

Eight of the eighteen (44\%) participants cited economic barriers to utilization of care services. Majority of these related to failure to find money to meet transport costs to the HIV care service points. Although services were provided free of cost to the patient at the clinics, some patients needed to travel long distances to the health unit. The long distance, coupled with lack of transport money, posed a major barrier to entry into HIV care. The participants referred to long distance to the clinics and high transport costs as the main barriers to entry into care. Three providers $(25 \%)$ agreed that transportation costs were a major hindrance to HIV care utilization. Below, we highlight some of the study participant economic barriers to care seeking.

I have a weak limb that became lame. I have to use a "boda boda" (motor cycle) for hire to move to all places. I had no money and failed to make it to the clinic. (IDI, Male, 20)

I failed to keep the clinic appointment because I am already sick and weak yet I do not have money to take me to the clinic neither do I have a relative to help them out of any problems. (IDI, Female, 22)

Some clients have failed because of the distance. When they imagine walking long distances and yet he or she is feeling unwell, they decide not to come. The problem is long distances, yet they do not have money to pay for transport. (KII, HIV counselor)

One orphaned male student attending a boarding school and taken care of by non-relatives who were unaware of his positive HIV status expressed his difficulty in accessing care:

I was not able to meet my appointment at the clinic because of lack of time and transport since I was far away at school. I attend a boarding school in a different district. When I go to school, they give me little pocket money, and this money was not going to be enough to cover my transport costs to the clinic. (IDI, Male, 17)

The fear of life-long HIV medication and its side effects prevented some individuals from entering care. The need for strict drug dosing schedules created a fear of failure to adhere to health worker recommendations. The perceptions about
HIV medications were principally based on reported experiences of other HIV-infected individuals who had received HIV medications.

Some people fear that once you begin on medication, it can accelerate death. However, it may be a wrong perception but I am not sure. Some people also complain that some drugs are hard to take. This scares some people away from HIV health care. (IDI, Female, 23)

One participant and one provider reported that the long waiting hours at the clinic were a barrier to entry into care. Patients were required to come to the clinic early, but it was not unusual for a patient to leave several hours later, especially if his/her clinic visit involved many activities. One participant narrated how the length of time spent at the clinic by some of his HIV-positive colleagues had dissuaded him from entering HIV care. The long waiting hours translated into lost work time and money.

Patients take the whole day there [at the clinic]; one may go there at 8:00 am and come back at 4:00 pm. This has not encouraged me to go to the clinic. (IDI, Male, 24) Many clients complain about the time they spend at Suubi. They say that you have to sacrifice the whole day and forego a lot of work; they don't care about life but rather time. The time they spend here is much and this delays them to enroll (KII, Female, Counselor)

\section{Perceived Susceptibility to HIV Disease}

A person's assessment of the likelihood of succumbing to HIV-related infections or death led to denial of HIV status. One study participant and one provider reported that doubts about the accuracy of HIV status were a barrier to enrollment for HIV care. One HIV-positive person had received HIV testing and been counseled but doubted his status because he was still in good health and physically strong, as reported below.

I feel strong and sometimes doubt the positive HIV test results. I also have a friend who told me yesterday that he has never had any sign or feeling of being unhealthy. He has been strong all the time, but the health workers say he has HIV. I feel reluctant after I doubt my HIV status. (IDI, Male, 25)

\section{Perceived Severity of HIV}

This concept is based on the HIV-positive person's judgment of how serious HIV infection and its consequences are to his/ 
her health. Feeling healthy was raised as a major reason for not seeking HIV medical care. The absence of physical symptoms diminished the need to seek care.

I did not feel sick. That is the main reason why I did not enroll. I will enroll at the clinic when I start falling sick or if I see any sign. (IDI, Female, 24)

\section{Discussion}

In a program which provided free access to community-based HIV care, $31.5 \%$ (361/1145) of persons who knew their HIV test results did not utilize HIV care. Young people between the ages of 15 and 24 years were significantly at higher risk of not utilizing care (Nakigozi et al. 2011). Thus, underutilization of HIV services among the young people remains a substantial problem in this rural setting.

This study explored barriers to enrollment for free HIV care among young people in Rakai, Uganda, using the HBM to assess factors affecting non-utilization of HIV care. Perceived barriers to using HIV care services included social concerns like the fear of stigma or the negative consequences of HIV status disclosure and economic barriers like long distance to the clinic and the high transport costs, long clinic waiting time, and fear of medication. Perceived susceptibility to HIV led to denial of HIV status while the absence of physical HIV symptoms was perceived to decrease the need for care. In this study, although HIV services were provided free at the respective clinics, we note that among these young persons, $44 \%$ of the study participants and $25 \%$ of the providers presented economic barriers as a hindrance to care utilization. These mainly related to lack of money to meet transport costs to the clinic and time and money lost during the long waiting hours at the clinic. These findings are similar to other studies that have shown that people with HIV frequently experience severe economic barriers to health care, which can prevent or delay diagnosis and treatment outcome (Richter et al. 2014). Transport is a known barrier for poor people to access HIV treatment and care (Mshana et al. 2006; Rosen et al. 2007; Tuller et al. 2010). In another study, among TBinfected patients, indirect cost of care constituted important determinants of poor treatment adherence, contributing to low cure rates and high risk of death among poor and vulnerable groups (Munro et al. 2007; Waitt and Squire 2011).

It is believed that economic support for patients can help overcome economic barriers to use of health services (Burström 2009; Peters et al. 2007; Qian et al. 2009). Although information on impact of economic strengthening on uptake of HIV care services is lacking, improved uptake of HIV screening and treatment and improved treatment outcomes have been reported in some social protection interventions, such as cash transfers (Boccia et al. 2011). In Uganda, studies on economic empowerment for adolescents showed that "asset building" reduced poverty, improved psychosocial functioning, and positively impacted attitudes and behaviors (Ssewamala et al. 2008). Also, interventions that incorporate economic opportunities may also help reduce the risk of HIV/AIDS in the next generation by reducing mental health problems and discouraging engagement in risky sexual behaviors (Ssewamala 2005; Ssewamala et al. 2008) and have positive effect on adolescents' self-rated health and mental health functioning (Ssewamala et al. 2009)

Findings from this study strongly highlight the importance of economic barriers to utilization of HIV care services and suggest the need to promote economic empowerment for HIVinfected young people, so as to promote access and adherence to HIV care.

\section{Study Strength and Limitations}

This study's strength lies in the use of qualitative methods and the inclusion of both patients' and HIV care providers' views. However, since the study was exploratory, the findings may not be generalizable. Also, due to logistical constraints, we interviewed 30 ( 18 non-users of care and 12 providers) participants who could be located and these may differ from those that were difficult to locate.

\section{Conclusion}

Our study suggests several barriers to enrollment into HIV care among adolescents and young adults aged 15-24 years. Economic barriers, particularly lack of transport money and loss of work hours due to long waiting time at the clinic, are highly prevalent as obstacles to utilization of care by this population, suggesting the need for economic strengthening for young persons living with HIV, so as to promote utilization of HIV care services. Stigma also remains a key barrier, and there is a need to provide youth-friendly services.

\section{Implications for Policy and Practice}

In order to promote HIV care utilization for HIV-infected young people, there is a need to integrate income generation in the HIV care package. HIV care providers should provide youth-friendly HIV care services.

Funding source This study was funded through a training grant from the Fogarty International Center, NIH grant number 2 D43 TW00150810, and, in part, by the African Doctoral dissertation fellowship award. SJR was supported by the Division of Intramural Research, National Institute of Allergy and Infectious Diseases, NIH. 


\section{References}

Althoff, K. N., Gange, S., Klein, M. B., Brooks, J. T., Hogg, R. S., Bosch, R. J., et al. (2010). Late presentation for human immunodeficiency virus care in the United States and Canada. Clinical Infectious Diseases, 50(11), 1512-1520.

Amuron, B., Namara, G., Birungi, J., Nabiryo, C., Levin, J., Grosskurth, H., et al. (2009). Mortality and loss-to follow-up during the pretreatment period in an antiretroviral therapy programme under normal health service conditions in Uganda. BMC Public Health, 9, 290

Bassett, I. V., Wang, B., Chetty, S., Mazibuko, M., Bearnot, B., Giddy, J., et al. (2009). Loss to care and death before antiretroviral therapy in Durban, South Africa. Journal of Acquired Immune Deficiency Syndromes, 51(2), 135-139.

Boccia, D., Hargreaves, J., Lönnroth, K., Jaramillo, E., \& Weiss, J. (2011). Cash transfer and microfinance interventions for tuberculosis control: review of the impact evidence and policy implications. The International Journal of Tuberculosis and Lung Disease, 15, S37-49.

Burström, B. (2009). Market-oriented, demand-driven health care reforms and equity in health and health care utilization in Sweden. International Journal of Health Services, 39, 271-285.

Fagan, J. L., Bertolli, J., \& McNaghten, A. D. (2010). Understanding people who have never received HIV medical care: a populationbased approach. Public Health Reports, 125(4), 520-527.

Graham, S. M., Masese, L., Gitau, R., Jalalian-Lechak, Z., Richardson, B. A., Peshu, N., et al. (2010). Antiretroviral adherence and development of drug resistance are the strongest predictors of genital HIV-1 shedding among women initiating treatment. Journal of Infectious Diseases, 202(10), 1538-1542.

Granich, R., Crowley, S., Vitoria, M., Smyth, C., Kahn, J. G., \& Bennett, R. (2010). Highly active antiretroviral treatment for the prevention of HIV transmission. Journal of the International AIDS Society, 13(1), 1.

Janz, N. K., \& Becker, M. H. (1984). The health belief model: a decade later. Health Education Quarterly, 11(1), 1-47.

Kamali, A., Seeley, J. A., Nunn, A. J., Kengeya-Kayondo, J. F., Ruberantwari, A., \& Mulder, D. W. (1996). The orphan problem: experience of a sub-Saharan Africa rural population in the AIDS epidemic. AIDS Care, 8(5), 509-15.

Losina, E., Bassett, I. V., Giddy, J., Chetty, S., Regan, S., \& Walensky, R. P. (2010). The "ART" of linkage: pre-treatment loss to care after HIV diagnosis at two PEPFAR sites in Durban, South Africa. PloS One, 5(3), e9538.

Marks, G., Gardner, L. I., Craw, J., \& Crepaz, N. (2010). Entry and retention in medical care among HIV-diagnosed persons: a metaanalysis. AIDS, 24(17), 2665-2678.

Mermin, J., Ekwaru, J. P., Liechty, C. A., Were, W., Downing, R., \& Ransom, R. (2006). Effect of co-trimoxazole prophylaxis, antiretroviral therapy, and insecticide treated bednets on the frequency of malaria in HIV-1-infected adults in Uganda: a prospective cohort study. The Lancet, 367(9518), 1256-1261.

Mkanta, W. N., \& Uphold, C. R. (2006). Theoretical and methodological issues in conducting research related to health care utilization among individuals with HIV infection. AIDS Patient Care and STDs, 20(4), 293-303.

Mshana, G. H., Wamoyi, J., Busza, J., Zaba, B., Changalucha, J., Kaluvya, S., et al. (2006). Barriers to accessing antiretroviral therapy in Kisesa, Tanzania: a qualitative study of early rural referrals to the national program. AIDS Patient Care and STDs, 20(9), 649-657.

Munro, S., Lewin, S., Smith, H., Engel, M., Fretheim, A., \& Volmink, J. (2007) Patient adherence to tuberculosis treatment: a systematic review of qualitative research. PLoS Med, 4: doi:10.1371/journal. pmed.0040238.
Nakigozi, G., Makumbi, F., Reynolds, S., Galiwango, R., Kagaayi, J., \& Nalugoda, F. (2011). Non-enrollment for free community HIV care: findings from a population-based study in Rakai, Uganda. AIDS Care, 23(6), 764-770.

Nakigozi, G., Atuyambe, L., Kamya, M., Makumbi, F. E., Chang, L. W., \& Nakyanjo, N. (2013). A qualitative study of barriers to enrollment into free HIV care: perspectives of never-in-care HIV-positive patients and providers in Rakai, Uganda. Biomed Research International, 2013, 470245.

Peters, D., Garg, A., Bloom, G., Walker, D., Brieger, W., \& Rahman, M. H. (2007). Poverty and access to health care in developing countries. Annals of the New York Academy, 1136, 161-171.

Qian, D., Pong, R., Yin, A., Nagarajan, K., \& Meng, Q. (2009). Determinants of health care demand in poor, rural China: the case of Gansu Province. Health Policy Planning, 24, 2324-334.

Richter, L. M., Lönnroth, K., Desmond, C., Jackson, R., Jaramillo, E., \& Diana, W. (2014). Economic support to patients in HIV and TB grants in rounds 7 and 10 from the global fund to fight AIDS, tuberculosis and malaria. PloS One, 9(1), e86225.

Rosen, S., Fox, M. P., \& Gill, C. J. (2007). Patient retention in antiretroviral therapy programs in sub-Saharan Africa: a systematic review. PLoS Med, 4(10), e298.

Samet, J. H., Freedberg, K. A., Stein, M. D., Lewis, R., Savetsky, J., \& Sullivan, L. (1998). Trillion virion delay: time from testing positive for HIV to presentation for primary care. Archives of Internal Medicine, 158(7), 734-740.

Sauerborn, R., Berman, P., \& Nougtara, A. (1996). Age bias, but no gender bias, in the intra-household resource allocation for health care in rural Burkina Faso. Health Transition Review, 6(2), 131-45.

Skovdal, M., Campbell, C., Madanhire, C., Nyamukapa, C., \& Gregson, S. (2011). Challenges faced by elderly guardians in sustaining the adherence to antiretroviral therapy in HIV-infected children in Zimbabwe. AIDS Care: Psychological and Socio-medical Aspects of AIDS/HIV, 23(8), 957-964.

Ssewamala, F. M. (2005). Children development accounts in Africa: a pilot study (CSD Working Paper No. 05-05). St. Louis: Washington University, Center for Social Development.

Ssewamala, F. M., Alicea, S., Bannon, W. M., \& Ismayilova, L. (2008). A novel economic intervention to reduce HIV risks among schoolgoing AIDS orphans in rural Uganda. Journal of Adolescent Health, 42, 102-104.

Ssewamala, F. M., Han, C. K., \& Neilands, T. B. (2009). Asset ownership and health and mental health functioning among AIDS-orphaned adolescents: findings from a randomized clinical trial in rural Uganda. Social Science \& Medicine, 69(2), 191-8.

Tuller, D. M., Bangsberg, D. R., Senkungu, J., et al. (2010). Transportation costs impede sustained adherence and access to HAART in a clinic population in southwestern Uganda: a qualitative study. AIDS Behavior 14(4), 778-84.

UNAIDS (2012). Global report: UNAIDS report on the global. AIDS epidemic 2012

UNAIDS. (2013). Global report: UNAIDS report on the global. AIDS epidemic 2013.

Waitt, C., \& Squire, S. (2011). A systematic review of risk factors for death in adults during and after tuberculosis treatment. The International Journal of Tuberculosis and Lung Disease, 15, 871885.

Weiser, S. D., Tsai, A. C., Gupta, R., et al. (2012) Food insecurity is associated with morbidity and patterns of healthcare utilization among HIV-infected individuals in a resource-poor setting. AIDS, 26(1), 67-75.

Weiss, M. G., Ramakrishna, J., \& Somma, D. (2006). Health-related stigma: rethinking concepts and interventions. Psychology, Health and Medicine, 11(3), 277-287. 